International Journal of Current Advanced Research

ISSN: O: 2319-6475, ISSN: P: 2319 - 6505, Impact Factor: SJIF: 5.995

Available Online at www.journalijcar.org

Volume 6; Issue 5; May 2017; Page No.3981-3984

DOI: http://dx.doi.org/10.24327/ijcar.2017.3984.0411

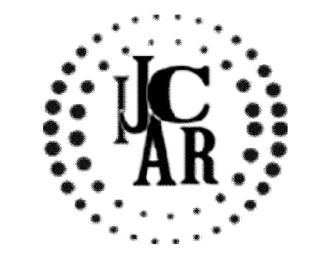

Research Article

\title{
A STUDY ON HIGH LEVEL AMINOGLYCOSIDE RESISTANT ENTEROCOCCI ISOLATED FROM URINARY TRACT INFECTION
}

\author{
Reena Rajan1., Amirtha C ${ }^{2}$., Mohana Soundaram K.M ${ }^{3}$ and Anandi $V^{4}$
}

${ }^{1}$ Department of Microbiology, Penang International Dental College, Vinayaka Mission's University, Salem, Tamil Nadu 2Department of Medicine, Rajah Muthiah Medical College, Annamalai University, Chidambaram, Tamil Nadu

${ }^{3}$ Department of Microbiology, IRT Perundurai Medical College, Erode, Tamil Nadu

${ }^{4}$ Department of Microbiology, Vinayaka Mission's Medical College, Karaikkal, Tamil Nadu

\section{A R T I C L E I N F O}

\section{Article History:}

Received $13^{\text {th }}$ February, 2017

Received in revised form $15^{\text {th }}$ March, 2017

Accepted $14^{\text {th }}$ April, 2017

Published online $28^{\text {th }}$ May, 2017

\section{Key words:}

High Level Aminoglycoside resistance, High Level Gentamicin Resistance, Minimum Inhibitory Concentration, Agar screen method

\begin{abstract}
A B S T R A C T
Enterococci infection have been treated with cell wall active agents in combination with an aminoglycoside as the synergic effect overcomes the intrinsic resistance exhibited by Enterococci. High level aminoglycoside resistance leads to synergy resistance and treatment failure.The present study was conducted to determine the prevalence of high level aminoglycoside resistance among isolates of enterococci from urine sampleA total of 147 Enterococci isolates were included in this study. High Level Aminoglycoside Resistance (HLAR) was determined by agar dilution method and E test. Of the total isolates, $81.63 \%$ were Enterococcus faecalis, $14.29 \%$ were Enterococcus faecium and $4.08 \%$ were Enterococcus avium. 60(40.82\%) were found to be resistant to Ampicillin, 76(51.70\%) to High LeveGentamicin (HLG), 24(16.33\%) to Nitrofurantoin by disk diffusion method. Out of 120 E.faecalis isolates, 39(32.50\%) were resistant to Ampicillin and 57(47.50\%) to High Level Gentamicin.Among the E.faecium isolates studied, $18(85.71 \%)$ were resistant to Ampicillin, 12(57.14\%) to High Level Gentamicin. High level gentamicin resistance (MIC $\geq 2000 \mu \mathrm{g} / \mathrm{ml}$ ) was seen in $22.45 \%$ isolates by agar dilution method. By E test $55 \%$ E.faecalis isolates and $71.43 \%$ E.faecium were with a Minimum Inhibitory Concentration(MIC) of $\geq 1024 \mu \mathrm{g} / \mathrm{ml}$ of Gentamicin.Monitoring of HLAR in Enterococci should be carried out by high content aminoglycoside disk and agar screen method.
\end{abstract}

Copyright $(2017$ Reena Rajan et al. This is an open access article distributed under the Creative Commons Attribution License, which permits unrestricted use, distribution, and reproduction in any medium, provided the original work is properly cited.

\section{INTRODUCTION}

Enterococci are gram positive facultative anaerobes, which have become increasingly important as hospital acquired pathogens. Enterococci are second most common causative agent of nosocomial urinary tract infection. Enterococci are responsible for health care associated infections such as blood stream, intraabdominal and surgical site infection. There has been a world wide trend in increasing occurrence of Enterococcal infection and emergence of antimicrobial resistance among these isolates during the past decade.[1]

Enterococci are intrinsically resistant to a number of antimicrobial agents and display low level resistance to aminoglycosides and lincosamides and their ability to acquire resistant genes to currently available antibiotics results in the selection and spread of Multi Drug Resistant (MDR) strains. Multi Drug Resistant Enterococci display a wide repertoire of drug resistant mechanisms such as overexpression of efflux pumps,

\section{*Corresponding author: Reena Rajan}

Department of Microbiology, Penang International Dental College, Vinayaka Mission's University, Salem,

Tamil Nadu drug target modification, inactivation of therapeutic agents and sophisticated cell envelope adaptive response that promotes survival of these organism in the human host and nosocomial environment. [1,2] The standard treatment for deep seated enterococci infections has been a bactericidal and synergistic combination of a cell wall acting agent (Ampicillin or Penicillin G) with an aminoglycoside (streptomycin /gentamicin).[2] Enterococci have acquired aminoglycoside resistance genes that encodes various aminoglycoside modifying enzymes, which result in high level resistance to aminoglycosides. The high level resistance to aminoglycoside abolishes synergism in the combination with betalactams and glycopeptides that are important in the treatment of severe enterococcal infection. $[3,4]$

The first gentamicin resistant strains was detected in the United states in 1981. High level gentamicin resistance is associated with bifunctional enzyme possessing acetylase (6') and phosphotransferase activities conferring resistance to all aminoglycosides except streptomycin.[4] High level streptomycin resistance may be mediated ribosomally or due to transferable plasmid encoding two aminoglycoside modifying enzyme streptomycin adenyl transferase and neomycin phosphotransferase. Most E.faecium isolates 
produce 6'acetylase transferase -acetylase that make them inherently resistant to amikacin, kanamycin, netilmycin and tobramycin there by nullifies the efficacy of combination therapy with these agents. $[4,5]$

Hence the present study was done to isolate, speciate and to determine the antimicrobial susceptibility profile of Enterococci isolates from urine sample and to detect the prevalence of high level aminoglycoside resistance in our clinical setting.

\section{MATERIALS AND METHODS}

A total of 147 isolates from clean catch mid stream urine were included in this study. The samples were cultured on Blood agar and Mac Conkey agar and incubated at $37^{\circ} \mathrm{C}$ for $18-24$ hours. Any significant growth obtained were identified by colony morphology, gram staining, catalase test, growth in $6.5 \%$ sodium chloride, aesculin hydrolysis in the presence of $40 \%$ bile and by biochemical reactions using conventional test scheme ( Facklam and Collins). Further identification to species level was done by using $1 \%$ carbohydrate solution of glucose, lactose, raffinose, arabinose, sorbose, sorbitol, sucrose, pyruvate utilisation test, arginine dihydrolase, motility and pigment production.[6]

\section{Antibiotic susceptibility testing}

Antimicrobial susceptibility testing was done on Muller Hinton agar by Kirby Bauer disk diffusion method using the following antibiotics: viz Ampicillin $(10 \mu \mathrm{g})$, Amikacin $(30 \mu \mathrm{g})$, Ciprofloxacin $(5 \mu \mathrm{g}), \quad$ Teicoplanin $\quad(30 \mu \mathrm{g})$, Nitrofurantoin $(300 \mu \mathrm{g})$, Kanamycin $(200 \mu \mathrm{g})$ and results were interpreted as per Clinical Laboratory Standard Institute (CLSI) guidelines.[7] Enterococcus faecalis 29212 was used as quality control strain. High level resistance aminoglycoside was determined by disc diffusion method using high level gentamicin disc $(120 \mu \mathrm{g})$ and streptomycin disc $(300 \mu \mathrm{g})$. A zone diameter of $\leq 6 \mathrm{~mm}$ was considered as resistant, $7-9 \mathrm{~mm}$ as intermediate and $\geq 10 \mathrm{~mm}$ as sensitive for kanamycin, gentamicin and streptomycin.[5,8]

Screening for aminoglycoside resistance was performed by using Brain heart infusion agar with $500 \mu \mathrm{g} / \mathrm{ml}$ for Gentamicin and $2000 \mu \mathrm{g} / \mathrm{ml}$ for Streptomycin. $10 \mu \mathrm{l}$ of bacterial suspension (equivalent to $0.5 \mathrm{McF}$ arland standard) was inoculated by spotting and the plates were incubated at $37^{0} \mathrm{C}$ for 24 hours. Presence of more than one colony or haze of growth was read as resistance. E.faecalis 51299 was used as positive control and E.faecalis 29212 was used as negative control [5]

Minimum Inhibitory concentration of Gentamicin was determined by $\mathrm{E}$ test and results were interpreted according to CLSI guidelines. MIC of $\geq 500 \mu \mathrm{g} / \mathrm{ml}$ for Gentamicin was considered as high level resistance. [9]

\section{RESULTS}

A total 147 Enterococci isolates obtained from urine sample were included in this study. Of the total isolates, $81.63 \%$ were Enterococcus faecalis, $14.29 \%$ were Enterococcus faecium and $4.08 \%$ were Enterococcus avium. Among the Enterococci studied, $60(40.82 \%)$ were found to be resistant to Ampicillin, 76(51.70\%) to High Level Gentamicin(HLG), $21(14.29 \%)$ to Teicoplanin, $99(67.35 \%)$ to Ciprofloxacin, $24(16.33 \%)$ to Nitrofurantoin by disk diffusion method.
Table 1 Overall resistance pattern of Enterococci by disk diffusion method

\begin{tabular}{cccc}
\hline Antibiotics & Sensitive & Intermediate & Resistant \\
\hline Ampicillin & $84(57.14 \%)$ & $3(2.04 \%)$ & $60(40.82 \%)$ \\
High Level & $110(74.83 \%)$ & $0(0.00 \%)$ & $37(25.17 \%)$ \\
Streptomycin & $42(28.57 \%)$ & $6(4.08 \%)$ & $99(67.35 \%)$ \\
Ciprofloxacin & $126(85.71 \%)$ & $0(0.00 \%)$ & $21(14.29 \%)$ \\
Teicoplanin & $27(18.37 \%)$ & $0(0.00 \%)$ & $120(81.63 \%)$ \\
Amikacin & $111(75.51 \%)$ & $12(8.16 \%)$ & $24(16.33 \%)$ \\
Nitrofurantoin & $71(48.30 \%)$ & $0(0.00 \%)$ & $76(51.70 \%)$ \\
High Level & $24(16.33 \%)$ & $0(0.00 \%)$ & $123(83.67 \%)$ \\
Gentamicin & & & \\
Kanamycin & &
\end{tabular}

Out of 120 E.faecalis isolates, 39(32.50\%) were resistant to Ampicillin, 57(47.50\%) to High Level Gentamicin, $18(15.00 \%)$ to Nitrofurantoin, $102(85.00 \%)$ to Kanamycin by Kirby Bauer disk diffusion method.

Among the E.faecium isolates studied, 18(85.71\%) were resistant to Ampicillin, 12(57.14\%) to HLG, $6(28.57 \%)$ to Nitrofurantoin and $18(85.71 \%)$ to Kanamycin Out of 6 E.avium isolates, 3 were resistant to Ampicillin and Kanamycin and 4 isolates to HLG. None of these isolates were resistant to Nitrofurantoin. [Table 2 ]

Table 2 Antibiotic resistant pattern of Enterococcus species by disk diffusion method

\begin{tabular}{cccc}
\hline Antibiotics & E.faecalis (120) & E.faecium (21) & E.avium (6) \\
\hline Ampicillin & $39(32.50 \%)$ & $18(85.71 \%)$ & $3(50.00 \%)$ \\
High Level & $30(25.00 \%)$ & $3(14.29 \%)$ & $4(66.67 \%)$ \\
Streptomycin & $78(65.00 \%)$ & $18(85.71 \%)$ & $3(50.00 \%)$ \\
Ciprofloxacin & $18(15.00 \%)$ & $3(14.29 \%)$ & $0(0.00 \%)$ \\
Teicoplanin & $99(82.50 \%)$ & $18(85.71 \%)$ & $3(50.00 \%)$ \\
Amikacin & $18(15.00 \%)$ & $6(28.57 \%)$ & $0(0.00 \%)$ \\
Nitrofurantoin & $57(47.50 \%)$ & $12(57.14 \%)$ & $4(66.67 \%)$ \\
High Level Gentamicin & $102(85.00 \%)$ & $18(85.71 \%)$ & $3(50.00 \%)$ \\
Kanamycin & & &
\end{tabular}

By agar dilution $103(70.07 \%)$ isolates showed high level gentamicin resistance with $\mathrm{MIC} \geq 500 \mu \mathrm{g} / \mathrm{ml}$ and $49(33.33 \%)$ were resistant to high level streptomycin (HLS) with MIC $\geq 2000 \mu \mathrm{g} / \mathrm{ml}$.[Table 3]

Table 3 Determination of HLGR among Enterococci by Agar dilution method

\begin{tabular}{cccc}
\hline \multirow{2}{*}{ Isolate } & \multicolumn{3}{c}{ HLGR } \\
\cline { 2 - 4 } & $\mathbf{5 0 0} \boldsymbol{\mu \mathbf { g } / \mathbf { m l }}$ & $\mathbf{1 0 0 0} \boldsymbol{\mu \mathbf { g }} / \mathbf{m l}$ & $\mathbf{2 0 0 0} \boldsymbol{\mu g} / \mathbf{m l}$ \\
\hline E.faecalis (120) & $84(70.00 \%)$ & $66(55.00 \%)$ & $21(17.50 \%)$ \\
E.faecium (21) & $15(71.43 \%)$ & $15(71.43 \%)$ & $9(42.86 \%)$ \\
E.avium (6) & $4(66.67 \%)$ & $4(66.67 \%)$ & $3(50.00 \%)$ \\
Total (147) & $103(70.07 \%)$ & $85(57.82 \%)$ & $33(22.45 \%)$ \\
\hline
\end{tabular}

HLGR: High Level Gentamicin Resistance

By E test $66(55.00 \%)$ E.faecalis isolates were resistant to High level Gentamicin $(\geq 1024 \mu \mathrm{g} / \mathrm{ml})$, HLGR was found to be $71.43 \%$ and $66.67 \%$ among E.faecium and E.avium isolates respectively.

\section{DISCUSSION}

Eventhough Enterococci are not regarded as highly pathogenic organisms, they are most commonly encountered in nosocomial bacteremia, endocarditis and urinary tract infection.[1] Enterococci with high level resistance to gentamicin are increasing in prevalence and are generally resistant to all aminoglycosides including amikacin, kanamycin, netilmycin and tobramycin with the occasional 
exception of streptomycin.[5] Kanamycin disc proves to be an accurate and reliable substitute to predict Amikacin-Penicillin synergy than does Amikacin. [4]

As routine disc diffusion does not detect High Level Aminoglycoside resistance among enterococci, MIC determination is definitive for high level resistance and resistance to synergism. Hence alternative methods such as agar screening, high content disc and broth dilution method have been proposed to detect HLAR among these strains. [2] In the present study, out of 147 isolates from urine sample, $81.63 \%$ were Enterococcus faecalis, $14.29 \%$ were Enterococcus faecium and $4.08 \%$ were Enterococcus avium. A study conducted by Chandrim et al [10] have reported an isolation rate of $42.86 \%$ E.faecalis, $36.51 \%$ E.faecium and $19.05 \%$ E.avium from urine sample. Out of these, $34 \%$ E.faecalis, 29\% E.faecium and 20\% E.avium were resistant to High Level Gentamicin by disk diffusion method.

In another Indian study Revati et al [11] have shown $56.86 \%$ E.faecalis and $33.33 \%$ E.faecium were isolated from urine. In contrast to present study, Maradia et al [12] have reported E.faecium $(67.95 \%)$ as the common species isolated from urine, followed by E.faecalis (32.05\%)

Among the Enterococci isolates studied, 60(40.82\%) were found to be resistant to Ampicillin, 76(51.70\%) to High Level Gentamicin (HLG), $99 \quad(67.35 \%)$ to Ciprofloxacin, $24(16.33 \%)$ to Nitrofurantoin by disk diffusion method.In a study among urinary isolates, Maradia et al [12] have reported 91.67\% Enterococci resistant to Ampicillin, 29.49\% to HLG and $76.28 \%$ resistant to Ciprofloxacin. HLGR(32\%) was reported in a study among clinical isolates of Enterococci by Archana et al. [13] A study among clinical isolates of Enterococci, by Bhatt et al [14] 53\% isolates were with high level resistance to gentamicin and 38\% showed high level resistance to streptomycin by disc diffusion method.

In the present study, out of 120 E.faecalis isolates, $39(32.50 \%)$ were resistant to Ampicillin, 57(47.50\%) to High Level Gentamicin and 30(25.00\%) to High Level Streptomycin by Kirby Bauer disk diffusion method. Among the E.faecium isolates studied, $18(85.71 \%)$ were resistant to Ampicillin, $12(57.14 \%)$ to HLG and 3(14.29\%) to HLS. A high percentage of High Level Aminoglycoside Resistance (HLAR) was found in E.faecium than in E.faecalis. In a study among urine sample by Amit et al [15], $33 \%$ of E. faecalis were HLGR and $31 \%$ were HLSR, where as $96.67 \%$ of E.faecium were reported to be HLGR and $90 \%$ as HLSR. A similar finding was also reported by N.Ganguide et al. [8] A study by Seema et al [16] have shown high level gentamicin resistance more common among enterococci isolated from urine sample $(41.50 \%)$ compared to other clinical samples.In another study, Sivasankari et al [17] have reported $48.70 \%$ E.faecalis and $54.60 \%$ E.faecium isolates resistant to High level gentamicin.

A study by Mendritta et al [18] have reported high level aminoglycoside resistance significantly higher among Enterococcus faecium than in E.faecalis with $46 \%$ isolates showed high level resistance to gentamicin and streptomycin by agar dilution method. In our study 103 (70.07\%) isolates showed high level gentamicin resistance with $\mathrm{MIC} \geq$ $500 \mu \mathrm{g} / \mathrm{ml}$ and $49(33.33 \%)$ were resistant to high level streptomycin with $\mathrm{MIC} \geq 2000 \mu \mathrm{g} / \mathrm{ml}$.
By E test $66(55.00 \%)$ E.faecalis isolates were resistant to High level Gentamicin $(1024 \mu \mathrm{g} / \mathrm{ml})$, HLGR was found to be $71.43 \%$ and $66.67 \%$ among E.faecium and E.avium isolates respectively. There was correlation between MIC for Gentamicin produced by agar dilution method and E-test. In the present study, $55 \%$ E.faecalis isolates and $71.43 \%$ E.faecium were with a Minimum Inhibitory Concentration of $1024 \mu \mathrm{g} / \mathrm{ml}$ for Gentamicin by $\mathrm{E}$ test. In a similar study K.Suresh et al [19] reported $48.27 \%$ E.faecalis and $6.90 \%$ E.faecium had MIC $>512 \mu \mathrm{g}$ to gentamicin by E test.

In a study among urinary isolates of Enterococci, Jyothi et al [20] showed $49 \%$ of isolates with high level resistance to gentamicin by Enz MIC strip method. In a South Indian study by Elango Padmini et al [21] among clinical isolates of enterococci $42.7 \%$ were HLGR (MIC $\geq 512 \mu \mathrm{g} / \mathrm{ml}$ ) by E test. In a study from North India by Jain et al [22] have reported high prevalence of HLAR among enterococci isolates from patients with bacteremia. $54 \%$ of the isolates were found to be HLAR and disc diffusion and agar screen method results for HLAR were concordant.

Enterococci are becoming increasingly resistant to traditional antimicrobial therapy. To treat complicated enterococcal infection, a bactericidal combination of penicillin (ampicillin or penicillin $\mathrm{G}$ with aminoglycoside (gentamicin or streptomycin) is required.However enterococcal resistance to high level aminoglycoside, ampicillin and vancomycin has resulted in limited therapeutic options. No treatment regime currently available is likely to produce reliable bactericidal effect on Enterococci with high level resistance to both gentamicin and streptomycin.

\section{CONCLUSION}

High Level Aminoglycoside Resistance (Gentamicin $\geq 500 \mu \mathrm{g} / \mathrm{ml}$ ) among Enterococci were found to be $70.07 \%$ in our study. The percentage resistance to high level aminoglycosides were higher among Enterococcus faecium than in Enterococcus faecalis. Results for HLAR detection by agar screen method and E test were concordant.Hence screening for enterococci for HLAR should be carried out by high content disk and agar screen method in clinical settings.

\section{References}

1. Barbara E.Murray 1990. The Life and Times of the Enterococcus. Clinical Microbiology Review, 3(1):4665

2. David J. Herman, Dalen. Gerding 1991. Antimicrobial Resistance among Enterococci Antimicrobial agents and Chemotherapy, 35(1): :1-4

3. A.H. C. Uttley, R. C. George, J. Naidoo, N. Woodford,A. P. Johnson, C. H. Collins, D. Morrison, A. J. Gilfillan L. E. Fitch, J.Heptonstall 1989 . Highlevel vancomycin-resistant enterococci causing hospital Infections. Epidemiology and Infection, 103(1).:173-181

4. Joseph.W.Chow. Aminoglycoside resistance in Enterococci 2000. Clinical Infectious Diseases, 31:586-9

5. Seema Sood, Meenakshi Malhotra, B K Das, Arti Kapil 2008. Enterococcal infections and antimicrobial resistance. Indian Journal of Medical Research, 128(2):111-121 
6. R.R.Facklam and M.D Collins 1989.Identification of Enterococcus species isolated from human infections by a conventional test scheme. Journal of Clinical microbiology, 27(4):731-745.

7. Clinical and Laboratory Standard Institute (CLSI). Performance standards for antimicrobial susceptibility testing. $18^{\text {th }}$ ed.Wayne USA .CLSI, 2008.

8. Nita Gangurde, Manisha Mane, Sunita Phatale 2014.Prevalence of Multidrug Resistant Enterococci in a Tertiary Care Hospital in India :A Growing Threat. Open Journal of Microbiology, 4(1):11-15

9. Martha .L. Sanchez, Mary S Barrett, Ronald N Jones 1992 .Use of E test to Predict High Level Resistance to Aminoglycosides Among Enterococci. Journal of Clinical Microbiology, 30(11):3030-3032

10. Chandrim Sengupta, Anusha Venkatesan, Sangamitra.V, Subha .S, Radha Madhavan 2015. High Level Gentamicin Resistance among Enterococcus species Isolated from Clinical Samples in a Tertiary care Hospital. International Journal of Medical and Health Sciences, 4(4):437-440

11. Revati Sharma, Chitra Pai 2013. Prevalence of Various Enterococcal infections and Its Antibiotic susceptibility with special reference to vancomycin and High Level Gentamicin resistance in a tertiary care centre in Navi Mumbai .International. Journal Of .Pharmaceutical Science .Review and Research, 23(2):122-125

12. Mitali R Maradia, Kanksha Mehta, Khyati Prajapati, Minesh Vadsmiya, Pranay Shah, Mahendra Vegad 2017. Prevalence of multidrug -resistant Enterococcus species isolated from urine samples in a tertiary care hospital, Western India. International Journal of Medical Science and Public Health, 6(4):1-5

13. Archana Rao .K, Deepa S, Venkatesha D 2014. Enterococci -A journey of a successful pathogen. International Archives of Integrated Medicine, 1(4):4957.

14. Bhatt P, Shete V, Sahni A K, Grover N, Chaudhari C N, Dudhat V L, Pandit P 2014. Prevalence of High level Aminoglycoside resistance in Enterococci at a Tertiary Care Centre. International Journal of Recent Scientific Research,5 (8) : 1515-1517
15. Amit Singh, Balram Ji Omar, R .C.Pande, Ramkant Yadhav, Amita Dohrae 2013. Speciation and Antimicrobial susceptibility pattern of Enterococcui from a rural tertiary health care center-A two year study. International Journal of Science and Technology,3(1):1-9

16. Seema Mittal, Pooja Singla, Antariksha Deep, Kiran Bala, Rama Sikka, Meenu Garg, and Uma Chaudhary 2016. Vancomycin and High Level Aminoglycoside Resistance in Enterococcus spp in a Tertiary Health Care Centre: A Therapeutic Concern. Journal of Pathogens, 1-5.

17. Sivasankari S, Somasunder V.M, Senthamarai S, Anitha C, Kumudhavathi M.S, Suneel Kumar Kumar Reddy A, Amshavathani S K, Venugopal 2013. Detection of High Level Aminoglycosides Resistant Enterococci In A Tertiary Care Hospital. International Organisation of Scientific Research Journal Of Pharmacy and Biological Sciences ,8(5) :53-57

18. DK Mendritta, H Kaur, V Deotale, DC Thamke, R Narang, P Narang 2008. Status of High level Aminoglycoside resistant Enterococcus faecium and Enterococcus faecalis in a rural hospital of Central India. Indian Journal of Medical Microbiology, 26(4):369-71

19. K.Suresh, B.Saipriya, G. Viswanath 2013. Isolation, Speciation and Determination of High level Aminoglycoside resistance of Enterococci among hospitalised patients in Davangere. National Journal of Laboratory Medicine, 2(1):12-15

20. Jyothi P, Metri B C, Peerapur B V 2014. High Level Resistance to Aminoglycosides in Urinary Isolates of Enterococci. Annals of Medical and Health Sciences Research ,4 (1):58

21. Elango Padmasini, R. Padmaraj, and S. Srivani Ramesh 2014. High Level Aminoglycoside Resistance and Distribution of Aminoglycoside Resistant Genes among Clinical Isolates of Enterococcus Species in Chennai, India. The ScientificWorld Journal ,1-5

22. Sarika Jain, Ashwani Kumar, Bineeta Kashyap, Iqbal R Kaur 2011. Clinico-epidemiological profile and highlevel aminoglycoside resistance in enterococcal septicemia from a tertiary care hospital in east Delhi. International Journal of Applied and Basic Medical Research,1(2): 80-83

\section{How to cite this article:}

Reena Rajan et al (2017) 'A Study On High Level Aminoglycoside Resistant Enterococci Isolated From Urinary Tract Infectionr', International Journal of Current Advanced Research, 06(05), pp. 3981-3984.

DOI: http://dx.doi.org/10.24327/ijcar.2017.3984.0411 